\title{
Application of Frequency Diversity to Suppress Grating Lobes in Coherent MIMO Radar with Separated Subapertures
}

\author{
Long Zhuang and Xingzhao Liu \\ Department of Electronic Engineering, Shanghai Jiao Tong University, Shanghai 200240, China \\ Correspondence should be addressed to Xingzhao Liu, xzhliu@sjtu.edu.cn
}

Received 5 June 2008; Revised 27 December 2008; Accepted 1 May 2009

Recommended by Ioannis Psaromiligkos

\begin{abstract}
A method based on frequency diversity to suppress grating lobes in coherent MIMO radar with separated subapertures is proposed. By transmitting orthogonal waveforms from $M$ separated subapertures or subarrays, $M$ receiving beams can be formed at the receiving end with the same mainlobe direction. However, grating lobes would change to different positions if the frequencies of the radiated waveforms are incremented by a frequency offset $\Delta f$ from subarray to subarray. Coherently combining the $M$ beams can suppress or average grating lobes to a low level. We show that the resultant transmit-receive beampattern is composed of the range-dependent transmitting beam and the combined receiving beam. It is demonstrated that the range-dependent transmitting beam can also be frequency offset-dependent. Precisely directing the transmitting beam to a target with a known range and a known angle can be achieved by properly selecting a set of $\Delta f$. The suppression effects of different schemes of selecting $\Delta f$ are evaluated and studied by simulation.
\end{abstract}

Copyright ( 2009 L. Zhuang and X. Liu. This is an open access article distributed under the Creative Commons Attribution License, which permits unrestricted use, distribution, and reproduction in any medium, provided the original work is properly cited.

\section{Introduction}

Unlike a traditional phased-array radar system, which can only transmit scaled versions of a single waveform, a multi-input multi-output (MIMO) radar system has shown much flexibility by transmitting multiple orthogonal (or incoherent) waveforms [1-15]. The waveforms can be extracted at the receiving end by a set of matched filters. Each of the extracted components contains the information of an individual transmit-receive path. According to the processing modes for using this information, the MIMO radars can be divided into two classes. One class is noncoherent processing to overcome the radar cross section (RCS) fluctuation of the target [1-3]. In this scheme, the transmitting antennas are separated from each other to ensure that a target is observed from different aspects. The other class is coherent processing [4-13], where the receiving antennas are closely spaced to avoid ambiguity. By using different phase shifts associated with different propagation paths, a better spatial resolution can be obtained. Some of the recent work on this class MIMO radar has been reviewed in [13].
A hybrid processing mode for MIMO radar with separated antennas has been proposed in [14, 15]. The authors pointed out that the locations of targets can be previously determined within a limited area by non-coherent processing. Then, by coherent processing the resolution can be improved to resolve targets located in the same range cell. It is demonstrated that by phase synchronizing across the sparse antennas, the resolution of MIMO radar can be improved to the level of the carrier wavelength $\lambda_{0}$. However, as also stated in $[14,15]$, the high resolution mode enabled by the coherent processing of sparse antennas creates grating lobes stemming from the large separation between antennas. To avoid ambiguity in target localization, it is necessary to suppress the unwanted grating lobes to a low level. Randomly positioning the antennas can break up the grating lobes at the cost of higher sidelobes $[16,17]$. The statistical analysis of sidelobes in coherent processing sparse MIMO radar with randomly positioned antennas has been studied in [18].

Inspired by using frequency diversity to suppress grating lobes in conventional sparse arrays [19-21], in this paper, we propose a frequency diverse method to suppress grating lobes 


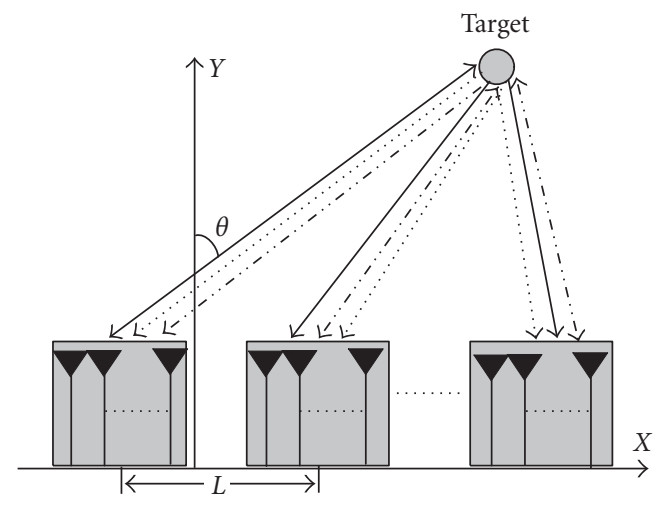

FIGURE 1: MIMO radar with separated subapertures.

in sparse MIMO aperture radar systems. We focus on monostatic sparse MIMO radar, that is, each antenna acts as both transmitter and receiver. Frequency diversity is achieved by transmitting orthogonal waveforms with diverse frequencies from each antenna simultaneously. The radiated frequencies are progressively incremented by a frequency offset $\Delta f$. By coherently combining the receiving beams formed at different frequencies, the grating lobes can be suppressed or averaged to a low level. It is shown that the transmit-receive beampattern (BP) of MIMO radar with frequency diversity is composed of the range-dependent transmitting beam and the combined receiving beam. The range-dependent beam has been studied in [22-24]. We demonstrate that the rangedependent beam can also be frequency offset-dependent. Precisely steering the transmitting beam can be achieved by properly selecting a set of frequency offsets.

The remainder of this paper is organized as follows. Section 2 describes the basic sparse MIMO aperture signal model. The BP of MIMO array with frequency diversity and the selection of the frequency offset are derived in Section 3. The simulation results are given in Section 4, and Section 5 is the conclusion.

\section{Basic Signal Model for Sparse Mimo Aperture Radar}

Consider a monostatic sparse MIMO aperture radar system with $M$ separated subarrays. Each subarray is a standard uniform linear array (ULA) with $N$ elements. Let $\mathbf{a}_{\text {sub }}(\theta)$ be the vector response of subarrays, where $\theta$ is the azimuth angle. For simplicity, suppose that the sparse distance $L$ between two adjacent subarrays is constant (Figure 1). We assume here that the target RCS is frequency independent. In the case of a single target at direction $\theta$ the signal received by the $m$ th subarray can be described as [7]

$$
\begin{gathered}
\mathbf{y}_{m}[k]=\alpha \sum_{i=1}^{M} \mathbf{A}_{i m}(\theta) \cdot \mathbf{s}_{i}[k]+\mathbf{w}_{m}[k], \\
m=1, \ldots, M, \quad k=1, \ldots, K,
\end{gathered}
$$

where $k$ is the time index, $\alpha$ is the complex amplitude of the received signal, $s_{i}[k]$ is the discrete form of the waveform transmitted by the $i$ th subarray, and $\mathbf{w}_{m}[k]$ is the additive noise at the $m$ th subarray. $\mathbf{A}_{i m}(\theta)$ reflects the phase shift from the $i$ th transmitting subarray to the $m$ th receiving subarray, that is,

$$
\mathbf{A}_{i m}(\theta)=\exp \left[-j 2 \pi f_{0}\left(\tau_{i}(\theta)+\tau_{m}(\theta)\right)\right], \quad i, m=1, \ldots, M,
$$

where $f_{0}$ is the operating frequency. For all the $M$ transmitted waveforms, there are $M \times M$ phase shifts in the receiving sparse array. By combining all the phase shifts, the sparse MIMO aperture array response can be written as

$$
\begin{aligned}
& \mathbf{A}(\theta)=\mathbf{a}(\theta) \mathbf{a}^{T}(\theta), \\
& \mathbf{a}(\theta)=\mathbf{a}_{\mathrm{sub}}(\theta) \otimes \mathbf{a}_{F}(\theta), \\
& \mathbf{a}_{F}(\theta)=\left[1, \exp \left(-j \frac{2 \pi f_{0}}{c} L \sin \theta\right), \ldots,\right. \\
&\left.\exp \left(-j \frac{2 \pi f_{0}}{c}(M-1) L \sin \theta\right)\right]^{T},
\end{aligned}
$$

where $(\cdot)^{T}$ denotes the transpose operator, $\otimes$ stands for the Kronecker operation, and $c$ is the speed of light.

In the matrix notation, (1) can be written as

$$
\mathbf{Y}[k]=\alpha \mathbf{A}(\theta) \mathbf{S}[k]+\mathbf{W}(k), \quad k=1, \ldots, K,
$$

where $\mathbf{Y}[k], \mathbf{S}[k]$, and $\mathbf{W}(k)$ are the received signal, the transmitted signal, and the additive noise, respectively.

If the output matrix $\mathrm{Y}$ in (4) is reorganized into a column vector, the sparse MIMO array response can be written as $\mathbf{a}_{\mathrm{MIMO}}(\theta)=\mathbf{a}(\theta) \otimes \mathbf{a}(\theta)$. The matched weight vector of the beamformer will be $\mathbf{a}_{\mathrm{MIMO}}\left(\theta_{0}\right)=\mathbf{a}\left(\theta_{0}\right) \otimes \mathbf{a}\left(\theta_{0}\right)$, where $\theta_{0}$ is the target direction of arrival (DOA). This gives rise to the following transmit-receive BP:

$$
\begin{aligned}
G_{\mathrm{MIMO}}(\theta) & =\left|\mathbf{a}_{\mathrm{MIMO}}^{H}\left(\theta_{0}\right) \mathbf{a}_{\mathrm{MIMO}}(\theta)\right|^{2} \\
& =\left|\left[\mathbf{a}^{H}\left(\theta_{0}\right) \otimes \mathbf{a}^{H}\left(\theta_{0}\right)\right][\mathbf{a}(\theta) \otimes \mathbf{a}(\theta)]\right|^{2} \\
& =\left|\mathbf{a}^{H}\left(\theta_{0}\right) \mathbf{a}(\theta)\right|^{2}\left|\mathbf{a}^{H}\left(\theta_{0}\right) \mathbf{a}(\theta)\right|^{2} \\
& =\left|\mathbf{a}^{H}\left(\theta_{0}\right) \mathbf{a}(\theta)\right|^{4},
\end{aligned}
$$

where $(\cdot)^{H}$ stands for the Hermitian operation.

The resultant transmit-receive $\mathrm{BP}$ can be viewed as the multiplication of the transmitting beam and the receiving beam $[7,8]$. In this context, the transmitting beam is identical with the receiving beam. Furthermore, the transmitreceive $\mathrm{BP}$ of the MIMO array is equivalent to the twoway $\mathrm{BP}$ of the conventional phased array. There are two differences between a MIMO radar array and a conventional phased array. First, the orthogonal waveforms in a MIMO radar array enable the radiated energy to cover a broad sector, and there is no scanning at the transmitting end. Second, the forming of the transmit beam in a MIMO radar array can be implemented at the receiving end by post-processing, and the transmit-receive BP is obtained using only the received signals. 
It can be seen from (5) that grating lobes still exist in the transmit-receive BP if the array configuration is sparse. Since the forming of the transmit beam is implemented at the receiving end, the grating lobes would not lead to energy leaking at the transmitting end, unlike the case in conventional sparse arrays. However, at the receiving end, the grating lobes may cause the ambiguity response to the targets outside the mainbeam direction. To eliminate this ambiguity, the grating lobes must be suppressed to a low level. In next section, a method based on frequency diversity is described to suppress grating lobes in sparse MIMO aperture radars.

\section{MIMO Radar with Frequency Diversity}

We call a MIMO radar array with frequency diversity a MIMO-FD array. The grating lobes suppression is achieved by coherently combining $M \times M$ different returns at the receiving end. The key is to utilize properly the phase differences between the returns with different transmit frequencies.

3.1. MIMO-FD Array Response. Assume that the frequency transmitted by the $i$ th transmitting subarray is $f_{i}=f_{0}+(i-$ 1) $\Delta f$, where $\Delta f$ is the frequency offset. For a point target at the range $r$ and angle $\theta$, the signal received by the $m$ th subarray can be written as

$$
\begin{gathered}
\mathbf{y}_{m}[k]=\alpha \sum_{i=1}^{M} \mathbf{B}_{i m}(r, \theta) \mathbf{s}_{i}[k]+\mathbf{w}_{m}[k], \\
m=1, \ldots, M, \quad k=1, \ldots, K,
\end{gathered}
$$

where $\mathbf{B}_{i m}(r, \theta)$ is the phase shift written as

$$
\begin{aligned}
\mathbf{B}_{i m}(r, \theta)= & \exp \left(-j 2 \pi f_{i}\left(\tau_{i}(\theta)+\tau_{m}(\theta)-\frac{2 r}{c}\right)\right) \\
= & \exp \left(-j 2 \pi f_{0}\left(\tau_{i}(\theta)+\tau_{m}(\theta)\right)\right) \\
\quad & \times \exp \left(-j 2 \pi(i-1) \Delta f\left(\tau_{i}(\theta)+\tau_{m}(\theta)\right)\right) \\
& \times \exp \left(j 2 \pi f_{i} \frac{2 r}{c}\right) .
\end{aligned}
$$

The first exponential term of (7) is the conventional phase shift and is the same as (2). The second exponential term shows an additional phase shift, which is dependent on the frequency offset. The third exponential term, which is rangedependent and is generally ignored for the single frequency processing, should be additionally processed.

Combining all the phase shifts, the MIMO-FD array response matrix can be written as

$$
\mathbf{B}(\theta)=\left[\begin{array}{c}
h\left(f_{1}, \theta\right) \otimes \mathbf{a}\left(f_{1}, \theta\right) \\
\vdots \\
h\left(f_{i}, \theta\right) \otimes \mathbf{a}\left(f_{i}, \theta\right) \\
\vdots \\
h\left(f_{M}, \theta\right) \otimes \mathbf{a}\left(f_{M}, \theta\right)
\end{array}\right]^{T}, \quad i=1,2, \ldots, M,
$$

with

$$
\begin{aligned}
h\left(f_{i}, \theta\right)= & \exp \left\{-j \frac{2 \pi}{c} f_{i}[(i-1) L \sin \theta-2 r]\right\}, \\
\mathbf{a}\left(f_{i}, \theta\right)= & \mathbf{a}_{\mathrm{sub}}\left(f_{i}, \theta\right) \otimes \mathbf{a}_{F}\left(f_{i}, \theta\right), \\
\mathbf{a}_{F}\left(f_{i}, \theta\right)= & {\left[1, \exp \left(-j \frac{2 \pi}{c} f_{i} L \sin \theta\right), \ldots,\right.} \\
& \left.\exp \left(-j \frac{2 \pi}{c} f_{i}(M-1) L \sin \theta\right)\right],
\end{aligned}
$$

where the exponential term $h\left(f_{i}, \theta\right)$ describes the phase shift caused by the waveform transmitted from the $i$ th subarray, the vector $\mathbf{a}\left(f_{i}, \theta\right)$ is the sparse array response for the $i$ th transmitted waveform, and $\mathbf{a}_{\text {sub }}\left(f_{i}, \theta\right)$ is the subarray response for the $i$ th transmitted waveform.

The $M \times M$ phase shifts in (8) can be used to form $M$ receiving beams with the same mainlobe direction. However, the directions of grating lobes are not the same with the $n$th occurring at the angle location $\sin \theta_{n}=n\left(\lambda_{i} / L\right)$. Note that the locations of the grating lobes are wavelength dependent, that is, the grating lobes tend to change their positions in the $M$ receiving beams formed at $M$ different frequencies. By combining the $M$ beams, the level of grating lobes can be reduced.

The resultant transmit-receive BP of MIMO-FD array can be written as

$$
\begin{aligned}
G_{\text {MIMO-FD }}(\theta) & \\
& =G_{T}(r, \theta) G_{\mathrm{R}}(\theta) \\
& =\frac{\left|\mathbf{b}^{H}\left(r, \theta_{0}\right) \mathbf{b}(r, \theta)\right|^{2}\left|\sum_{i=1}^{M} \mathbf{a}^{H}\left(f_{i}, \theta_{0}\right) \mathbf{a}\left(f_{i}, \theta\right)\right|^{2}}{M^{2}} .
\end{aligned}
$$

The detailed derivation of (10) is shown in Appendix A. Compared with (5), the transmit-receive BP of the MIMOFD array can also be treated as the multiplication of the transmitting beam and the receiving beam. The left term in the numerator of (10) represents the transmitting beam. It should be noted that the diverse frequencies across the sparse array will cause the beam direction to be rangedependent. Other terms in (10) represent the combination of the individual beams formed at different frequencies. The grating lobe suppression effect depends on such parameters as the frequency offset $\Delta f$, the number of transmitted waveforms $M$, and the sparse distance $L$. A larger $\Delta f$ leads to larger movement of grating lobes, more transmitted waveforms mean more beams can be combined at the receiving end to reduce grating lobes, and different sparse distances result in different locations and numbers of grating lobes. The relationship between the frequency offset and the ratio of the Peak Sidelobe Level (PSL) to the Average Sidelobe Level (ASL) is given in Appendix B.

However, the direction of the transmitting beam $G_{T}(r, \theta)$ is range-dependent. The range-dependent beam has been studied in [22-24] with the characteristic that the beam direction is not constant but varies with range. Therefore, 


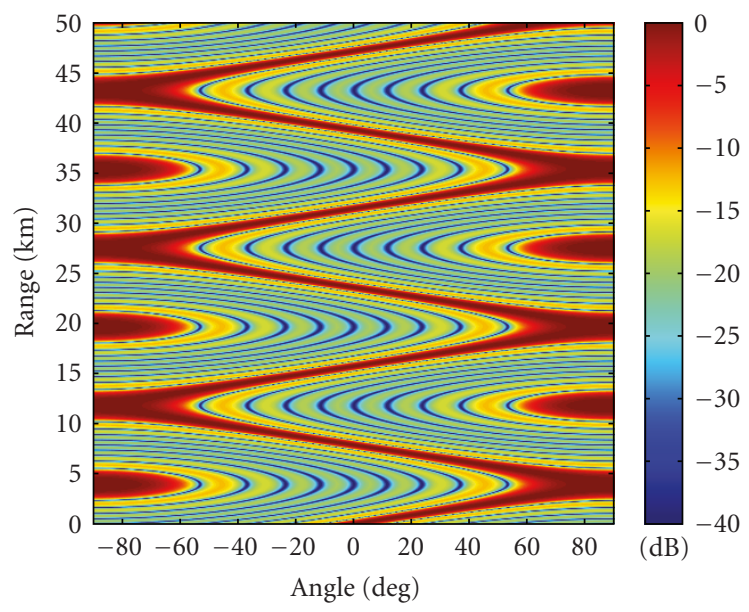

Figure 2: The beam direction varies as a function of range.

in the MIMO-FD context, the apparent angle of the transmittingbeam $G_{T}(r, \theta)$ is not necessarily equal to that of the receiving beam $G_{\mathrm{R}}(\theta)$ at certain ranges. If the transmitting beam is desired to be directed to a known target at $(r, \theta)$, the frequency offset must be deliberately selected to keep the direction consistent with that of the receiving beam.

3.2. Frequency Offset-Dependent Beam. Let $\theta^{\prime}$ denote the apparent angle of the transmitting beam. Then, the relationship between the apparent angle and the nominal angle can be written as $[22,23]$

$$
\theta^{\prime}=\arcsin \left(\sin \theta-\frac{\Delta f \cdot 2 r}{f_{0} \cdot L}+\frac{\Delta f \cdot \sin \theta}{f_{0}}\right)
$$

It should be noted that $2 r$ in (11) indicates the round-trip distance, which is different from that in $[22,23]$. Assume the nominal angle $\theta=0$ and the antenna spacing $L=\lambda_{0} / 2$. Then, the apparent angle can be written as

$$
\theta^{\prime}=\arcsin \left(\frac{4 r \Delta f}{c}\right)
$$

The above equation demonstrates that for a known nominal angle, if $\Delta f$ is fixed, the beam direction is a function of range $r$. Such beams are called range-dependent beams. However, if range $r$ is fixed, the beam direction is a function of $\Delta f$. Such beams can be defined as frequency offsetdependent beams.

3.3. Examples of Frequency Offset-Dependent Beam. The range dependence is first examined for a 10-element standard ULA with $\Delta f=30 \mathrm{kHz}$ and $f_{0}=10 \mathrm{GHz}$. Figure 2 depicts that the beam varies in the range dimension. Note that there exists $\pi$ ambiguity, and the beam is directed at angle $0^{0}$ only at certain ranges.

The frequency offset-dependent beam for a target at range $r=50 \mathrm{~km}$ is depicted in Figure 3. The beam direction varies with frequency offset from 0 to $30 \mathrm{kHz}$. A 1-D cut of the beam directed at $\left(50 \mathrm{~km}, 0^{0}\right)$ with different frequency

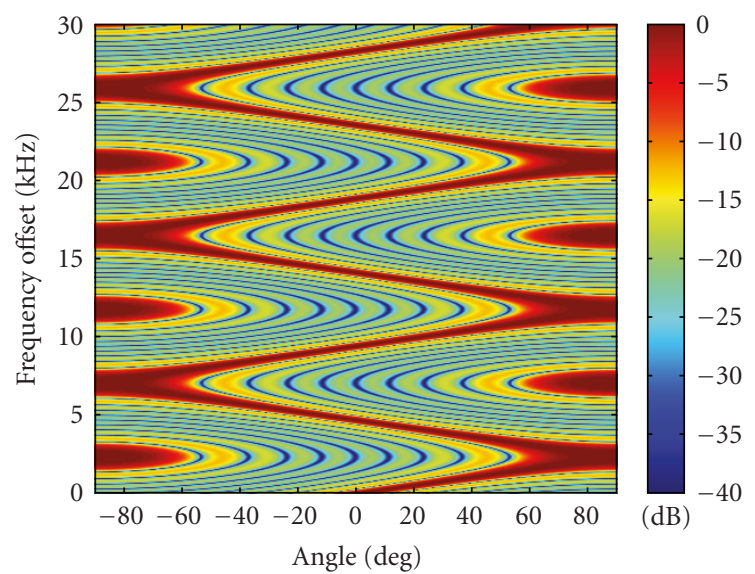

FIgURE 3: The beam direction varies as a function of frequency offset.

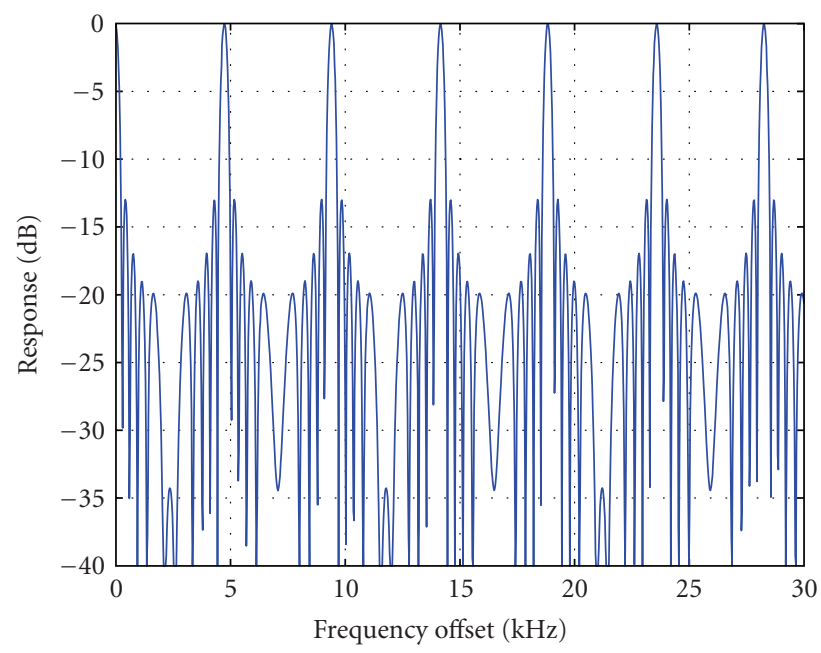

FIGURE 4: The beam directs to a target at $\left(50 \mathrm{~km}, 0^{0}\right)$ with different frequency offsets.

offsets is shown in Figure 4. The beam is repeatedly directed to the target with some certain frequency offsets, which can provide additional freedom to choose the frequency offset.

An analytic expression of the frequency offset-dependent beam directed to the target at $(r, \theta)$ can be derived. According to (11), letting the apparent angle equal the nominal angle with $\pi$ ambiguity, we obtain

$$
\sin \theta^{\prime}=n \pi+\sin \theta-\frac{\Delta f}{f_{0}} \frac{2 r}{L}+\frac{\Delta f}{f_{0}} \sin \theta, \quad n=0,1, \ldots .
$$

Then the frequency offset is

$$
\Delta f=n \pi \frac{f_{0}}{2 r / L-\sin \theta}, \quad n=0,1, \ldots .
$$

3.4. Orthogonal Waveforms. To separate $M$ radiated waveforms at the receiving end or minimize the interference 


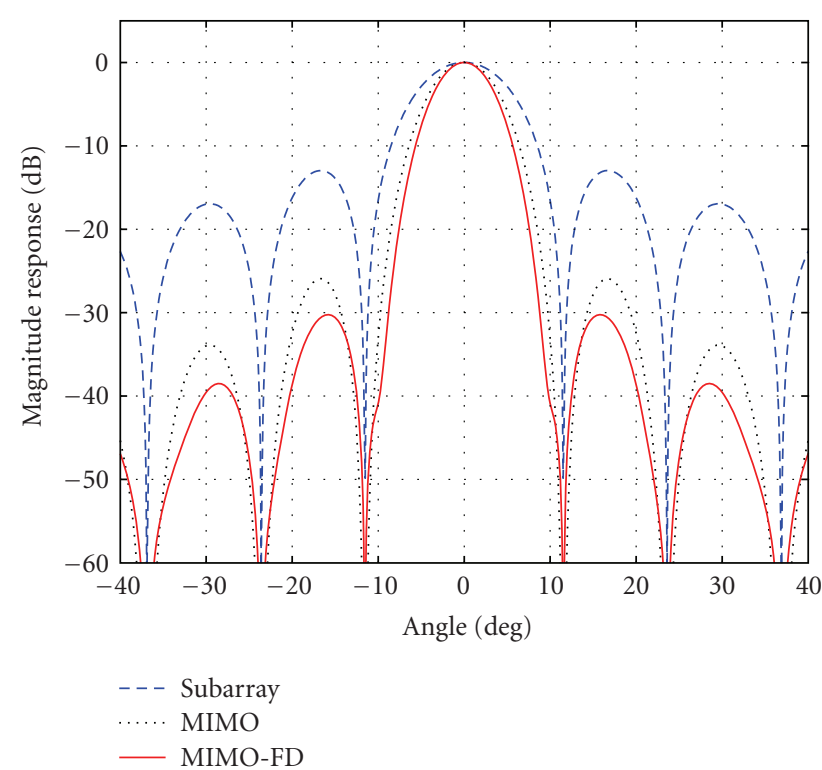

FIGURE 5: Beam pattern for MIMO and MIMO-FD arrays with half a wavelength spacing.

between waveforms, the correlation of two waveforms must satisfy

$$
s_{i}(t) * s_{m}^{H}(t)= \begin{cases}\delta(t), & i=m, \\ 0, & i \neq m,\end{cases}
$$

where $*$ denotes the convolution operator.

If the waveform duration is $T$, the cross-correlation of two waveforms is

$$
\begin{aligned}
& \int_{-T / 2}^{T / 2} s_{i}(t) s_{m}^{\dagger}(t) d t \\
&=\frac{1}{T} \int_{-T / 2}^{T / 2} \exp \left[j 2 \pi\left(f_{0}+(i-1) \Delta f\right) t\right] \\
& \quad \times \exp \left[-j 2 \pi\left(f_{0}+(m-1) \Delta f\right) t\right] d t \\
&=\operatorname{sinc}[\pi(i-m) \Delta f \cdot T],
\end{aligned}
$$

where $(\dagger)$ is the complex conjugate operator. So, to make waveforms orthogonal to each other, $\Delta f$ should satisfy

$$
\Delta f=\frac{n}{T}, \quad n=1,2, \ldots
$$

The waveforms can coexist if the frequency offset is $n / T$ between two subarray waveforms, that is, the orthogonality of waveforms can be achieved by separating the frequencies of waveforms by an integer multiple of the reciprocal of the waveform pulse duration.

3.5. Selection of Frequency Offset. The frequency offset of a MIMO-FD array should satisfy not only (14) to control the transmitting beam direction, but also (17) to make the transmitted waveforms orthogonal. Therefore, the frequency

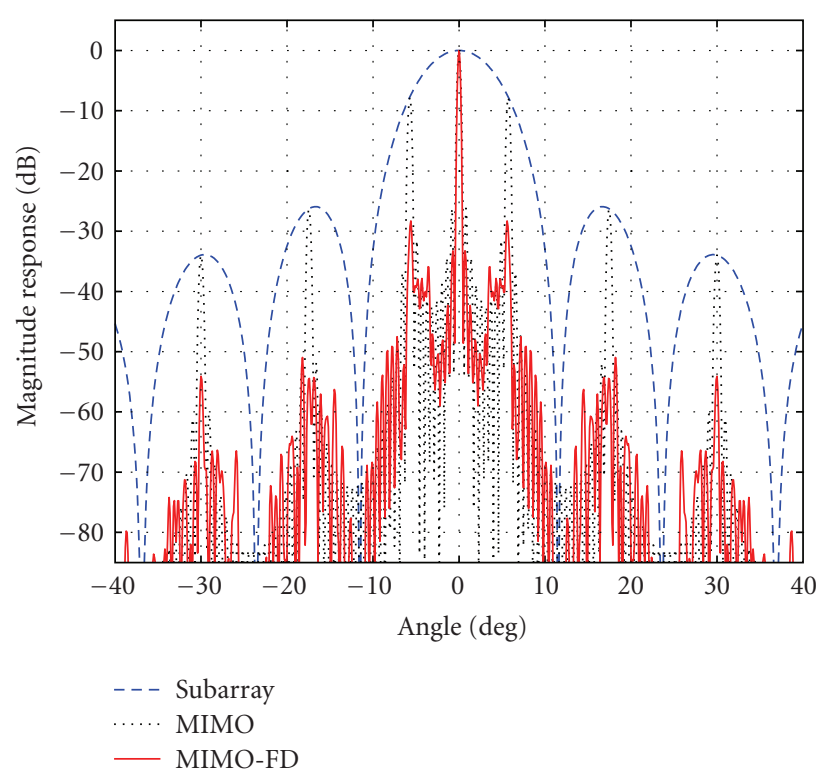

Figure 6: Beam pattern for MIMO and MIMO-FD arrays with the sparse distance $L=20 \lambda_{0} / 2$.

offset $\Delta f$ should be a multiple of the least common multiple (LCM) of (14), (17), that is,

$$
\Delta f=n \cdot \operatorname{LCM}\left[\frac{\pi f_{0}}{2 r / L-\sin \theta}, \frac{1}{T}\right], \quad n=1,2, \ldots .
$$

For comparison, consider a standard ULA with 10 elements. Suppose that the operating frequency is $10 \mathrm{GHz}$, and the signal duration is $T=1 \mu \mathrm{s}$. If the beam is desired to be directed to a target at $\left(35 \mathrm{~km}, 0^{0}\right)$, the set of $\Delta f$ can be selected as

$$
\Delta f \approx n \cdot 67 \mathrm{MHz}, \quad n=1,2, \ldots,
$$

according to (18).

Figure 5 shows the transmit-receive BPs for the MIMO and the MIMO-FD cases, where the frequency offset is $\Delta f=134 \mathrm{MHz}$. Compared with the phased-array radar, the MIMO array decreases the beamwidth by a factor of $\sqrt{2}$ [7]. The peak sidelobe level (PSL) of the MIMO array is about $-26.4 \mathrm{~dB}$, almost twice that of the phased array. The PSL drops even further to nearly $-30 \mathrm{~dB}$ for the MIMO-FD array. This demonstrates the effect of the frequency diversity in sidelobe reduction.

\section{Simulation Results}

In this section, the method to suppress grating lobes based on frequency diversity in coherent MIMO radar with separated subarrays addressed in Section 3 is evaluated by simulation. There are 10 subarrays, and each subarray is a 10-element ULA. The operating frequency is $10 \mathrm{GHz}$. The duration of each waveform pulse is $1 \mu \mathrm{s}$, the target is located at $\left(35 \mathrm{~km}, 0^{0}\right)$. We change the sparse distance and the frequency offset to test the suppression effect. 


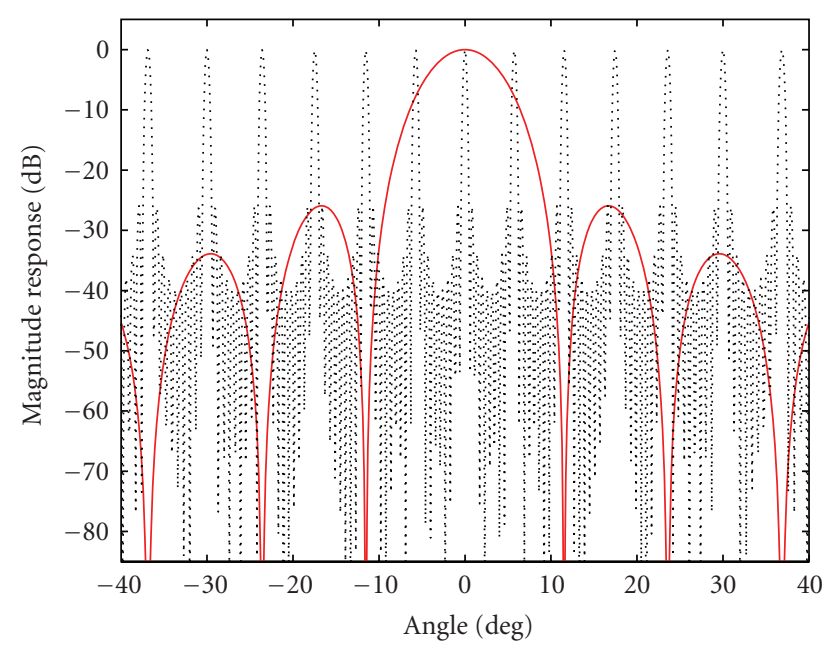

FIGURE 7: Grating lobes cancelled by the nulls of the subarray beam with $M=10$ and $L=20 \lambda_{0} / 2$.

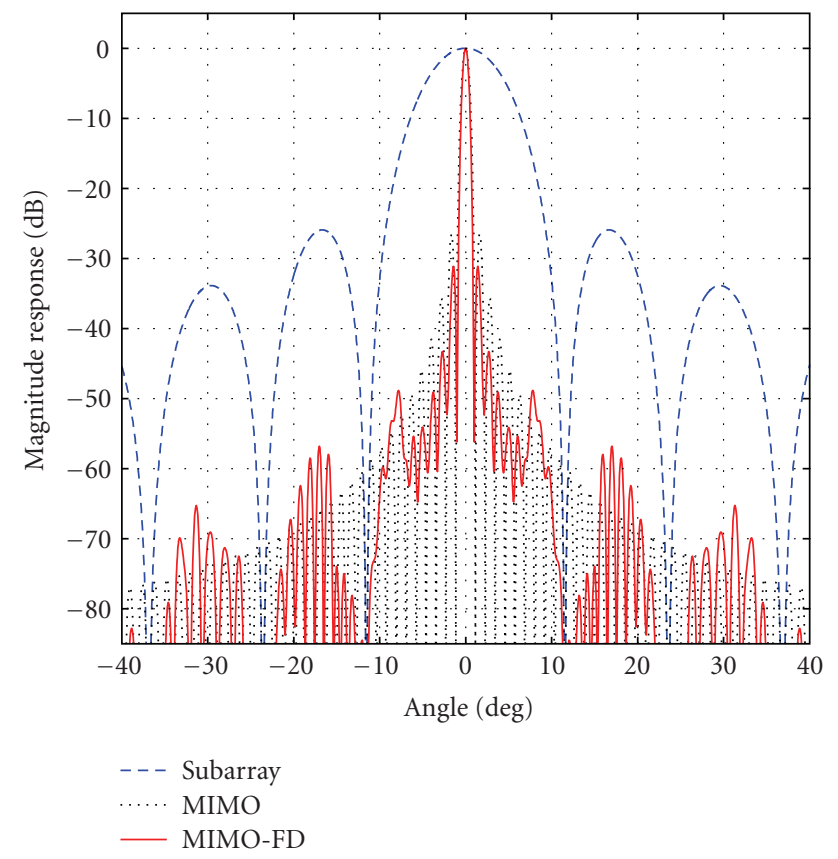

Figure 8: Beam pattern for MIMO and MIMO-FD arrays with the sparse distance $L=10 \lambda_{0} / 2$.

First suppose that the sparse distance between subarrays is $L=20 \cdot\left(\lambda_{0} / 2\right)$. As described in Section 3, the frequency offset can be set as $\Delta f=134 \mathrm{MHz}$. Figure 6 depicts the transmit-receive BP. It can be seen that there exist high grating lobes in the MIMO BP. However, the PSL is reduced to nearly $-28.5 \mathrm{~dB}$ with frequency diverse waveforms transmitted. It should be noted that the subarray beam has two important effects on the transmit-receive BP. The first is that it functions as an amplitude filter. The envelope of the MIMO $\mathrm{BP}$ is just the same as the subarray beam. The second is to cancel out some certain grating lobes using its nulls.

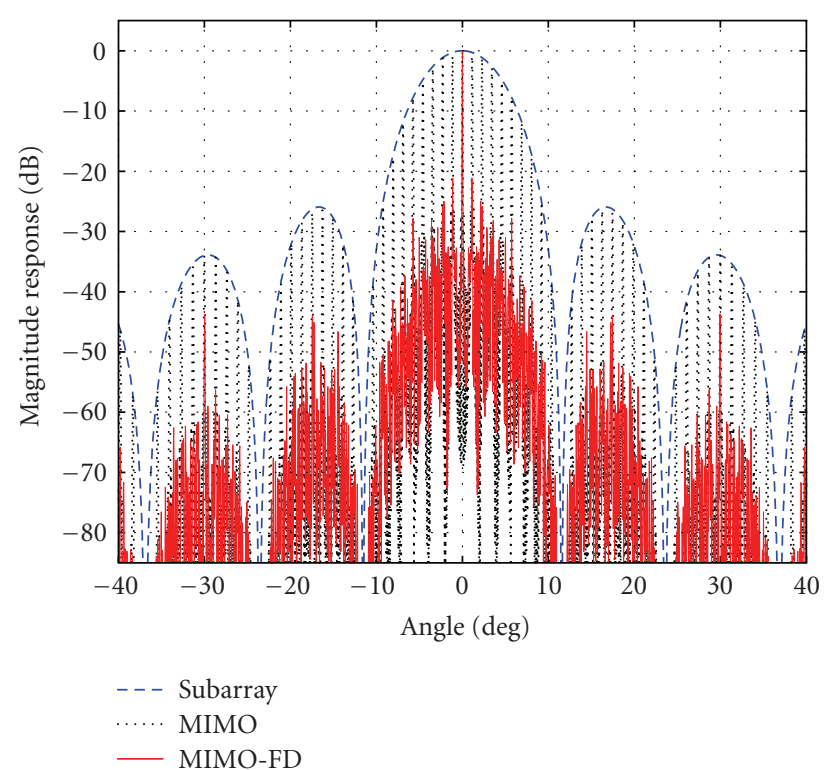

Figure 9: Beam pattern for MIMO and MIMO-FD arrays with the sparse distance $L=100 \lambda_{0} / 2$.

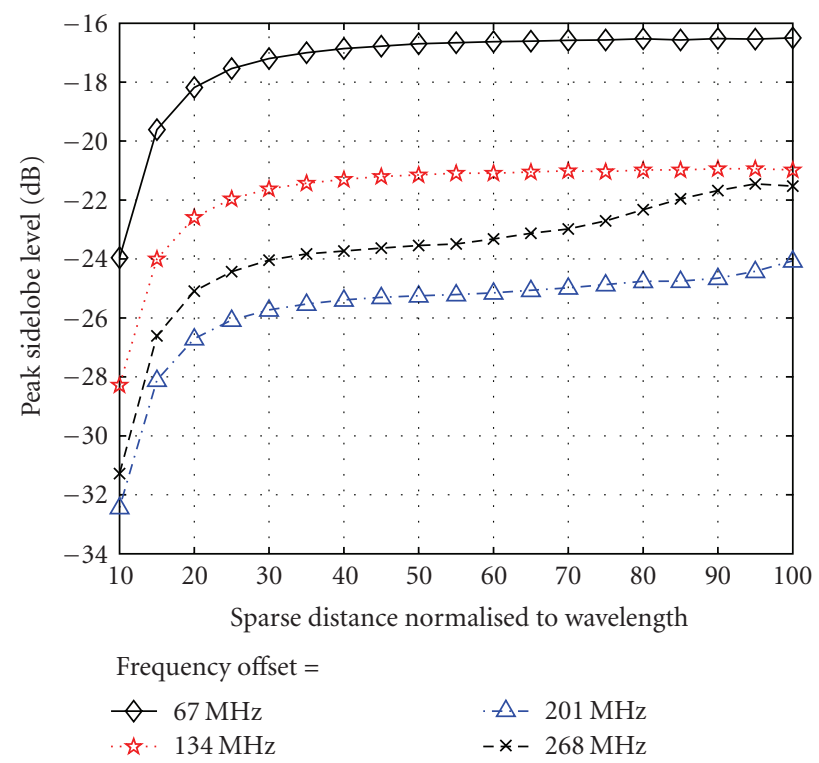

FIGURE 10: The PSL versus different sparse distances and frequency offsets.

In fact, for an ULA with the sparse distance $L=20$. $\left(\lambda_{0} / 2\right)$, there exist twelve grating lobes within the angle interval $\left[-40^{0}, 40^{\circ}\right]$. However, only six remain in either the MIMO or the MIMO-FD BP (see Figure 6). The reason is that the null locations of the subarray beam are just the same as some certain locations of grating lobes. This is shown in Figure 7. If the number of the nulls in the subarray beam is just equal to the number of grating lobes, the grating lobes can be totally cancelled out in whether the MIMO or the MIMO-FD BP. In this case, the element number of each subarray is equal to the sparse distance normalized to half a 


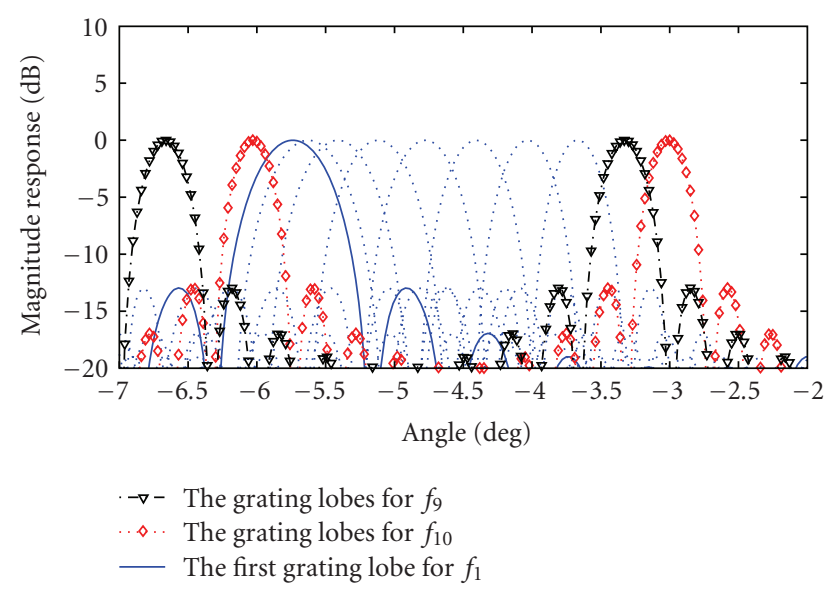

Figure 11: The different locations of grating lobes before combining, $\Delta f=201 \mathrm{MHz}, L=20 \lambda_{0} / 2$.

wavelength. So, we set the sparse distance as $L=10 \cdot\left(\lambda_{0} / 2\right)$, and the cancellation result is demonstrated in Figure 8 . The PSL for the MIMO BP is $-26.4 \mathrm{~dB}$, and it is the same as that of a filled MIMO array. Note that a lower PSL for MIMO-FD $\mathrm{BP}$ (nearly $-31 \mathrm{~dB}$ ) is achieved.

We further test the suppression effect for an even larger sparse distance $L=100\left(\lambda_{0} / 2\right)$. The result is depicted in Figure 9. The suppression effect is degraded with the PSL improved to $-21 \mathrm{~dB}$. The reason is that the receiving beams formed at different frequencies exhibit similar properties only in the region of mainlobe and neighboring sidelobes. Furthermore, for a fixed subarray number and a fixed subarray size, the larger the sparse distance is, the more the number of grating lobes in the beam is. With more neighboring grating lobes exhibiting similar properties, the suppression effect surely becomes worse.

It is evident that a larger frequency offset is helpful in suppressing grating lobes. Though the receiving beams formed at different frequencies exhibit similar properties in the mainlobe region, the locations of grating lobes become progressively different with the frequency offset increasing. When all the $M$ receiving beams are combined, the portion of the mainlobe region remains unchanged, and in the sidelobe region, the peaks will reduce to a lower level for a larger $\Delta f$.

There exists an upper limit to the frequency offset $\Delta f$ to achieve the optimum suppression effect. For the movement of the $n$th grating lobe by angle $\Delta \theta$, the transmit wavelength should be changed by $\Delta \lambda$ with $\Delta \theta=\sin ^{-1}\left(n \lambda_{0} / L\right)-$ $\sin ^{-1}\left(n \lambda_{i} / L\right) \approx n \Delta \lambda / L$. If the $(n+1)$ th grating lobe for the wavelength $\lambda_{i}$ moves into the $3-\mathrm{dB}$ beamwidth of the $n$th grating lobe for the wavelength $\lambda_{1}$, some additional energy will remain in this location. Thus, the suppression effect will be degraded. In Figure 10, we compare the suppression effects for different $\Delta f$ with the given simulation parameters. The sparse distance is changed in the interval $\left[20\left(\lambda_{0} / 2\right), 200\left(\lambda_{0} / 2\right)\right]$. Evidently, a better grating lobe suppression effect can be achieved using a larger frequency

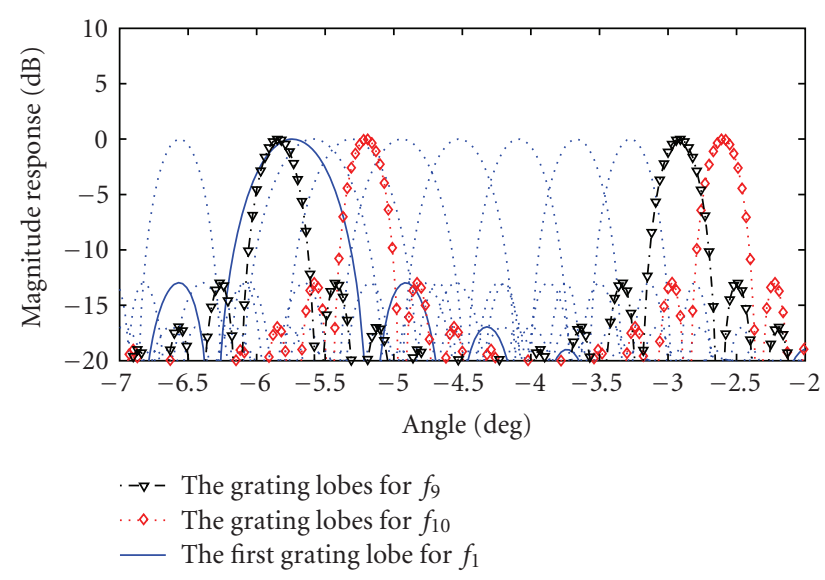

Figure 12: The different locations of grating lobes before combining, $\Delta f=268 \mathrm{MHz}, L=20 \lambda_{0} / 2$.

offset. However, the suppression effect gets worse for $\Delta f=$ $268 \mathrm{MHz}$ than for $\Delta f=201 \mathrm{MHz}$. This is interpreted in Figures 11, 12, which depict the beams formed at different frequencies before the coherent combining. It can be seen that with $\Delta f=201 \mathrm{MHz}$, the 2 nd grating lobe for the frequency $f_{10}$ is near in the $3-\mathrm{dB}$ beamwidth of the 1 st grating lobe for the frequency $f_{1}$. However, when $\Delta f$ is larger than $268 \mathrm{MHz}$, the 2 nd grating lobe for the frequency $f_{9}$ moves into the $3-\mathrm{dB}$ beamwidth of the 1st grating lobe for the frequency $f_{1}$. Since the grating lobes are mixed, the suppression effect will surely get worse.

\section{Conclusion}

A method based on frequency diversity to suppress grating lobes in sparse MIMO aperture radar is proposed in this paper. By the frequency diversity across the transmitting array, the locations of grating lobes in the receiving beams are totally changed. Coherently combining the $M$ receiving beams formed at different frequencies can suppress grating lobes to a low level. The resultant transmit-receive BP is composed of the range-dependent transmitting beam and the combined receiving beam. We demonstrate that, even though the transmitting beam is range-dependent, the beam can be precisely steered to a given target by deliberately selecting a set of $\Delta f$. The simulation results show that with a properly selected frequency offset, the method is effective in suppressing grating lobes in sparse MIMO aperture radars.

\section{Appendix}

\section{A. Deriving the Transmit-Receive BP of MIMO-FD Array}

Let $\phi=(4 \pi r / c)$, and let $\varphi=-(2 \pi / c) L \sin \theta$, then the MIMO-FD array response can be rewritten as 


$$
\begin{aligned}
\mathbf{B}(\theta) & =\left(\begin{array}{cccc}
\mathbf{a}_{\text {sub }}\left(f_{1}, \theta\right) \exp \left(j f_{1} \phi\right) & \mathbf{a}_{\text {sub }}\left(f_{1}, \theta\right) \exp \left(j f_{1}(\varphi+\phi)\right) & \cdots & \mathbf{a}_{\text {sub }}\left(f_{1}, \theta\right) \mathcal{A} \\
\mathbf{a}_{\text {sub }}\left(f_{2}, \theta\right) \exp \left(j f_{2}(\varphi+\phi)\right) & \mathbf{a}_{\text {sub }}\left(f_{2}, \theta\right) \exp \left(j f_{2}(2 \varphi+\phi)\right) & \cdots & \mathbf{a}_{\text {sub }}\left(f_{2}, \theta\right) \mathcal{B} \\
\vdots & \vdots & \cdots & \vdots \\
\mathbf{a}_{\text {sub }}\left(f_{M}, \theta\right) \exp \left(j f_{M}((M-1) \varphi+\phi)\right) & \mathbf{a}_{\text {sub }}\left(f_{M}, \theta\right) \exp \left(j f_{M}(M \varphi+\phi)\right) & \cdots & \mathbf{a}_{\text {sub }}\left(f_{M}, \theta\right) \mathcal{C}
\end{array}\right), \\
& =\mathbf{B}_{1}(\theta) \odot \mathbf{B}_{2}(\theta), \\
\mathcal{A} & =\exp \left(j f_{1}((M-1) \varphi+\phi)\right), \quad \mathcal{B}=\exp \left(j f_{2}(M \varphi+\phi)\right), \quad \mathcal{C}=\exp \left(j f_{M}(2(M-1) \varphi+\phi)\right),
\end{aligned}
$$

where $\odot$ represents the Hadamard product, and

$$
\begin{gathered}
\mathbf{B}_{1}(\theta)=\left[\begin{array}{cccc}
\exp \left(j f_{1} \phi\right) & \exp \left(j f_{1} \phi\right) & \cdots & \exp \left(j f_{1} \phi\right) \\
\exp \left(j f_{2}(\varphi+\phi)\right) & \exp \left(j f_{2}(\varphi+\phi)\right) & \cdots & \exp \left(j f_{2}(\varphi+\phi)\right) \\
\vdots & \vdots & \cdots & \vdots \\
\exp \left(j f_{M}((M-1) \varphi+\phi)\right) & \exp \left(j f_{M}((M-1) \varphi+\phi)\right) & \cdots & \exp \left(j f_{M}((M-1) \varphi+\phi)\right)
\end{array}\right], \\
\mathbf{B}_{2}(\theta)=\left[\begin{array}{cccc}
\mathbf{a}_{\mathrm{sub}}\left(f_{1}, \theta\right) \cdot 1 & \mathbf{a}_{\mathrm{sub}}\left(f_{1}, \theta\right) \cdot \exp \left(j f_{1} \varphi\right) & \cdots & \mathbf{a}_{\mathrm{sub}}\left(f_{1}, \theta\right) \cdot \exp \left(j f_{1}(M-1) \varphi\right) \\
\mathbf{a}_{\mathrm{sub}}\left(f_{2}, \theta\right) \cdot 1 & \mathbf{a}_{\mathrm{sub}}\left(f_{2}, \theta\right) \cdot \exp \left(j f_{2} \varphi\right) & \cdots & \mathbf{a}_{\mathrm{sub}}\left(f_{2}, \theta\right) \cdot \exp \left(j f_{2}(M-1) \varphi\right) \\
\vdots & \vdots & & \vdots \\
\mathbf{a}_{\mathrm{sub}}\left(f_{M}, \theta\right) \cdot 1 & \mathbf{a}_{\mathrm{sub}}\left(f_{M}, \theta\right) \cdot \exp \left(j f_{M} \varphi\right) & \cdots & \mathbf{a}_{\mathrm{sub}}\left(f_{M}, \theta\right) \cdot \exp \left(j f_{M}(M-1) \varphi\right)
\end{array}\right] .
\end{gathered}
$$

It is worthwhile to notice that $\mathbf{B}_{1}(\theta)$ can be viewed as the transmitting array response. $\mathbf{B}_{2}(\theta)$ represents the receiving array response with different frequencies transmitted from the same subarray. By joining the matrix $\mathbf{B}(\theta)$ into an $M M \times 1$ vector, the MIMO-FD array response vector can be written as

$$
\mathbf{a}_{\mathrm{MIMO}-\mathrm{FD}}(\theta)=\mathbf{b}(r, \theta) \otimes \mathbf{I}_{M \times 1} \odot \mathbf{a}(f, \theta),
$$

where $\mathbf{I}_{M \times 1}$ is an $M \times 1$ length identity vector, and

$$
\begin{aligned}
\mathbf{b}(r, \theta)= & {\left[\exp \left(j f_{1} \phi\right), \exp \left(j f_{2}(\varphi+\phi)\right), \ldots,\right.} \\
& \left.\exp \left(j f_{M}((M-1) \varphi+\phi)\right)\right],{ }^{T} \\
\mathbf{a}(f, \theta)= & {\left[\mathbf{a}\left(f_{1}, \theta\right), \ldots, \mathbf{a}\left(f_{i}, \theta\right), \ldots, \mathbf{a}\left(f_{M}, \theta\right)\right]^{T}, }
\end{aligned}
$$

where
The matched weight vector of the beamformer can be $\mathbf{a}_{\text {MIMO-FD }}\left(\theta_{0}\right)=\mathbf{b}\left(r, \theta_{0}\right) \otimes \mathbf{I}_{M \times 1} \odot \mathbf{a}\left(f, \theta_{0}\right)$, and the resultant transmit-receive BP is

$$
\begin{aligned}
G_{\text {MIMO-FD }}(\theta)= & \left|\mathbf{a}_{\text {MIMO-FD }}^{H}\left(\theta_{0}\right) \mathbf{a}_{\text {MIMO-FD }}(\theta)\right|^{2} \\
= & \left|\left(\mathbf{b}\left(r, \theta_{0}\right) \otimes \mathbf{I}_{M \times 1}\right)^{H}\left(\mathbf{b}(r, \theta) \otimes \mathbf{I}_{M \times 1}\right)\right|^{2} \\
& \times\left|\mathbf{a}^{H}\left(f, \theta_{0}\right) \mathbf{a}(f, \theta)\right|^{2} \\
= & \left|\mathbf{b}^{H}\left(r, \theta_{0}\right) \mathbf{b}(r, \theta)\right|^{2} \\
& \times\left|\frac{1}{M} \sum_{i=1}^{M} \mathbf{a}^{H}\left(f_{i}, \theta_{0}\right) \mathbf{a}\left(f_{i}, \theta\right)\right|^{2} \\
= & G_{T}(r, \theta) G_{\mathrm{R}}(\theta)
\end{aligned}
$$

with

$\mathbf{a}\left(f_{i}, \theta\right)=\mathbf{a}_{\text {sub }}\left(f_{i}, \theta\right) \otimes\left[1, \exp \left(j f_{i} \varphi\right), \ldots, \exp \left(j f_{i}(M-1) \varphi\right)\right]$.

$$
\begin{aligned}
G_{T}(r, \theta) & =\left|\mathbf{b}^{H}\left(r, \theta_{0}\right) \mathbf{b}(r, \theta)\right|^{2}, \\
G_{\mathrm{R}}(\theta) & =\left|\frac{1}{M} \sum_{i=1}^{M} \mathbf{a}^{H}\left(f_{i}, \theta_{0}\right) \mathbf{a}\left(f_{i}, \theta\right)\right|^{2} .
\end{aligned}
$$




\section{B. The Relationship between PSL/ASL and $\Delta f$}

Since the grating lobe suppression effect is achieved by coherently combining the $M$ receiving beams formed at different frequencies, the impact of subarray beam is omitted here. Though the beams formed at different frequencies exhibit similar properties in the mainlobe region, the correlation in the remainder part progressively decreases. The manner in which the region of sidelobes decorrelates with frequency can be calculated from the cross correlation function of two beams formed at two different frequencies [20].

Each receiving beam can be written as

$$
\begin{aligned}
F_{i}(\theta) & =\sum_{m=1}^{M} \exp \left(-j \frac{2 \pi}{c} f_{i} L(m-1)\left(\sin \theta-\sin \theta_{0}\right)\right) \\
& =\sum_{m=1}^{M} \exp \left(j f_{i}(m-1)\left(\varphi-\varphi_{0}\right)\right)
\end{aligned}
$$

where $\varphi_{0}=-(2 \pi / c) L \sin \theta_{0}$. The cross-correlation function of the two receiving beams formed at two subsequent frequencies is

$$
\begin{aligned}
& R_{i, i+1}=E\left\{F_{i}(\theta) F_{i+1}^{\dagger}(\theta)\right\} \\
& =E\left\{\sum_{m=1}^{M} \exp \left(j f_{i}(m-1)\left(\varphi-\varphi_{0}\right)\right)\right. \\
& \left.\times \sum_{n=1}^{M} \exp \left(-j f_{i+1}(n-1)\left(\varphi-\varphi_{0}\right)\right)\right\} \\
& =\sum_{m=1}^{M} \sum_{n=1}^{M} E\left\{\exp \left(j f_{i}(m-1)\left(\varphi-\varphi_{0}\right)\right)\right. \\
& \left.\times \exp \left(-j f_{i+1}(n-1)\left(\varphi-\varphi_{0}\right)\right)\right\} \\
& =E\left\{\sum_{\substack{m=1 \\
n=m}}^{M} \exp \left(-j \Delta f(m-1)\left(\varphi-\varphi_{0}\right)\right)\right\} \\
& +E\left\{\sum_{m=1}^{M} \exp \left(j f_{i}(m-1)\left(\varphi-\varphi_{0}\right)\right)\right\} \\
& \times E\left\{\sum_{\substack{n=1 \\
n \neq m}}^{M} \exp \left(-j f_{i+1}(n-1)\left(\varphi-\varphi_{0}\right)\right)\right\} \\
& =M \frac{\sin \left[\Delta f\left(\varphi-\varphi_{0}\right) / 2\right]}{\Delta f\left(\varphi-\varphi_{0}\right) / 2} \\
& +M(M-1) \frac{\sin \left[f_{i}\left(\varphi-\varphi_{0}\right) / 2\right]}{f_{i}\left(\varphi-\varphi_{0}\right) / 2} \\
& \frac{\sin \left[f_{i+1}\left(\varphi-\varphi_{0}\right) / 2\right]}{f_{i+1}\left(\varphi-\varphi_{0}\right) / 2}
\end{aligned}
$$

where $E\{\cdot\}$ is the expected value of $\{\cdot\}$. For a sparse array $M \ll L / \lambda_{i}$ and a high $f_{i} \gg \Delta f$, the second term is much smaller than the first one in the sidelobe region. Then

$$
R_{i, i+1} \cong M \frac{\sin \left[\Delta f\left(\varphi-\varphi_{0}\right) / 2\right]}{\Delta f\left(\varphi-\varphi_{0}\right) / 2} .
$$

The two beams are decorrelated in the sidelobe region when $\Delta f\left(\varphi-\varphi_{0}\right) / 2=\pi$. And so

$$
\begin{aligned}
\Delta f & =\frac{2 \pi}{\left(\varphi-\varphi_{0}\right)}=\frac{c}{(M-1) L\left(\sin \theta-\sin \theta_{0}\right)} \\
& =\frac{\lambda_{0} f_{0}}{(M-1) L\left(\sin \theta-\sin \theta_{0}\right)} .
\end{aligned}
$$

In addition, the ratio of the Peak Sidelobe Level (PSL) to the Average Sidelobe Level (ASL) of a linear random sparse array is approximately [16]

$$
\mathrm{PSL} / \mathrm{ASL} \cong \ln \frac{S\left(1+\left|\sin \theta_{0}\right|\right)}{\lambda_{0}},
$$

where $S$ is the array aperture length. If the sparse array is uniformly distributed, $S=(M-1) L$. In this case, the $\mathrm{PSL} / \mathrm{ASL}$ is

$$
\mathrm{PSL} / \mathrm{ASL} \cong \ln \frac{(M-1) L\left(1+\left|\sin \theta_{0}\right|\right)}{\lambda_{0}} .
$$

Combining (B.4) and (B.6) we can obtain

$$
\frac{\mathrm{PSL}}{\mathrm{ASL}} \cong \ln \left(\frac{f_{0}}{\Delta f} \cdot \frac{1+\left|\sin \theta_{0}\right|}{\sin \theta-\sin \theta_{0}}\right) .
$$

Variation of the frequency offset $\Delta f$ does not alter the ASL. Hence, the PSL can be reduced to get closer to the ASL with a larger $\Delta f$.

\section{References}

[1] E. Fishler, A. Haimovich, R. Blum, D. Chizhik, L. Cimini, and R. Valenzuela, "MIMO radar: an idea whose time has come," in Proceedings of the IEEE National Radar Conference, pp. 7178, Philadelphia, Pa, USA, April 2004.

[2] E. Fishler, A. Haimovich, R. Blum, L. Cimini, D. Chizhik, and R. Valenzuela, "Performance of MIMO radar systems: advantages of angular diversity," in Proceedings of the 38th Asilomar Conference on Signals, Systems and Computers (ACSSC '04), vol. 1, pp. 305-309, Pacific Grove, Calif, USA, November 2004.

[3] E. Fishler, A. Haimovich, R. S. Blum, L. Cimini Jr., D. Chizhik, and R. A. Valenzuela, "Spatial diversity in radarsmodels and detection performance," IEEE Transactions on Signal Processing, vol. 54, no. 3, pp. 823-838, 2006.

[4] V. F. Mecca, D. Ramakrishnan, and J. L. Krolik, "MIMO radar space-time adaptive processing for multipath clutter mitigation," in Proceedings of the 4th IEEE Sensor Array and Multichannel Signal Processing Workshop (SAM '06), pp. 249253, Waltham, Mass, USA, July 2006.

[5] P. Stoica, J. Li, and Y. Xie, "On probing signal design for MIMO radar," IEEE Transactions on Signal Processing, vol. 55, no. 8, pp. 4151-4161, 2007. 
[6] D. W. Bliss and K. W. Forsythe, "Multiple-input multipleoutput (MIMO) radar and imaging: degrees of freedom and resolution," in Proceedings of the 37th Asilomar Conference on Signals, Systems and Computers (ACSSC '03), vol. 1, pp. 54-59, Pacific Grove, Calif, USA, November 2003.

[7] I. Bekkerman and J. Tabrikian, "Target detection and localization using MIMO radars and sonars," IEEE Transactions on Signal Processing, vol. 54, no. 10, pp. 3873-3883, 2006.

[8] F. C. Robey, S. Coutts, D. Weikle, J. C. McHarg, and K. Cuomo, "MIMO radar theory and experimental results," in Proceedings of the 38th Asilomar Conference on Signals, Systems and Computers (ACSSC'04), vol. 1, pp. 300-304, Pacific Grove, Calif, USA, November 2004.

[9] J. Li, P. Stoica, L. Xu, and W. Roberts, "On parameter identifiability of MIMO radar," IEEE Signal Processing Letters, vol. 14, no. 12, pp. 968-971, 2007.

[10] H. Yan, J. Li, and G. Liao, "Multitarget identification and localization using bistatic MIMO radar systems," EURASIP Journal on Advances in Signal Processing, vol. 2008, Article ID 283483, 8 pages, 2008.

[11] D. R. Fuhrmann and G. S. Antonio, "Transmit beamforming for MIMO radar systems using partial signal correlation," in Proceedings of the 38th Asilomar Conference on Signals, Systems and Computers (ACSSC'04), vol. 1, pp. 295-299, Pacific Grove, Calif, USA, November 2004.

[12] L. Xu and J. Li, "Iterative generalized-likelihood ratio test for MIMO radar," IEEE Transactions on Signal Processing, vol. 55, no. 6, pp. 2375-2385, 2007.

[13] J. Li and P. Stoica, "MIMO radar with colocated antennas," IEEE Signal Processing Magazine, vol. 24, no. 5, pp. 106-114, 2007.

[14] N. H. Lehmann, A. M. Haimovich, R. S. Blum, and L. Cimini, "High resolution capabilities of MIMO radar," in Proceedings of the 40th Asilomar Conference on Signals, Systems and Computers (ACSSC '06), pp. 25-30, Pacific Grove, Calif, USA, November 2006.

[15] A. M. Haimovich, R. S. Blum, and L. Cimini, "MIMO radar with widely separated antennas," IEEE Signal Processing Magazine, vol. 25, no. 1, pp. 116-129, 2008.

[16] B. D. Steinberg, "The peak sidelobe of the phased array having randomly located elements," IEEE Transaction on Antenna and Propagation, vol. 20, no. 2, pp. 129-137, 1972.

[17] M. G. Bray, D. H. Werner, D. W. Boeringer, and D. W. Machuga, "Optimization of thinned aperiodic linear phased arrays using genetic algorithms to reduce grating lobes during scanning," IEEE Transactions on Antennas and Propagation, vol. 50, no. 12, pp. 1732-1742, 2002.

[18] M. A. Haleem and A. M. Haimovich, "On the distribution of ambiguity levels in MIMO radar," in Proceedings of the 42nd Asilomar Conference on Signals, Systems and Computers (ACSSC '08), Pacific Grove, Calif, USA, November 2008.

[19] M. Skolnik, "Resolution of angular ambiguities in radar array antennas with widely-spaced elements and grating lobes," IEEE Transaction on Antenna and Propagation, vol. 10, no. 3, pp. 351-352, 1962.

[20] B. D. Steinberg and E. H. Attia, "Sidelobe reduction of random arrays by element position and frequency diversity," IEEE Transactions on Antennas and Propagation, vol. 31, no. 6, pp. 922-930, 1983.

[21] D. R. Kirk, J. S. Bergin, P. M. Techau, and J. E. Don Carlos, "Multi-static coherent sparse aperture approach to precision target detection and engagement," in Proceedings of the IEEE Radar Conference, pp. 579-584, Arlington, Va, USA, May 2005.
[22] P. Antonik, M. C. Wicks, H. D. Griffiths, and C. J. Baker, "Frequency diverse array radars," in Proceedings of the IEEE Radar Conference, pp. 215-217, Verona, NY, USA, April 2006.

[23] P. Antonik, M. C. Wicks, H. D. Griffiths, and C. J. Baker, "Range dependent beamforming using element level waveform diversity," in Proceedings of the International Waveform Diversity and Design Conference, Lihue, Hawaii, USA, January 2006.

[24] P. Baizert, T. B. Hale, M. A. Temple, and M. C. Wicks, "Forward-looking radar GMTI benefits using a linear frequency diverse array," Electronics Letters, vol. 42, no. 22, pp. 1311-1312, 2006. 